\title{
Real-Time Rough Refraction
}
Charles de Rousiers ${ }^{1,3}$
Adrien Bousseau ${ }^{2,3}$
Kartic Subr ${ }^{1}$
Nicolas Holzschuch ${ }^{1}$
Ravi Ramamoorthi ${ }^{3}$
${ }^{1}$ INRIA Rhone-Alpes / LJK
${ }^{2}$ INRIA Sophia-Antipolis
${ }^{3}$ University of California, Berkeley

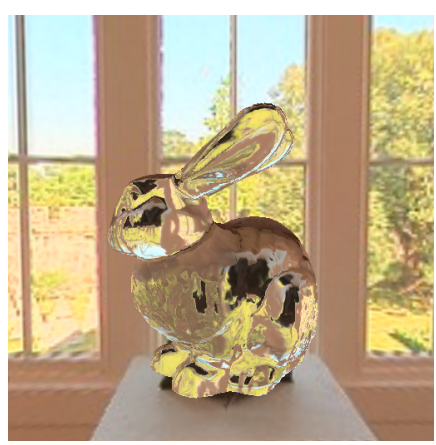

(a) $\kappa_{\gamma}^{D}=20000$

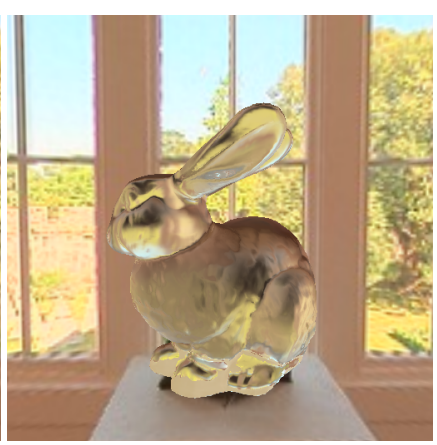

(b) $\kappa_{\gamma}^{D}=90$

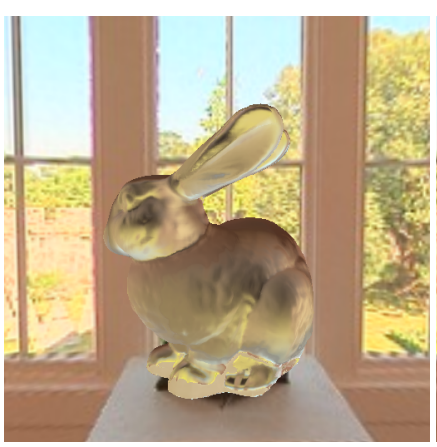

(c) $\kappa_{\gamma}^{D}=24$

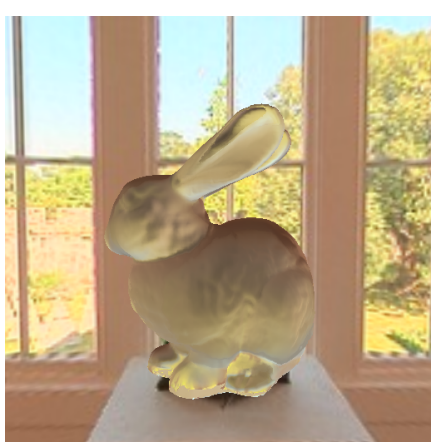

(d) $\kappa_{\gamma}^{D}=6.4$

Figure 1: The Bunny model rendered with a transparent material taking into account the scattering from rough surfaces, at the entering and exiting interfaces. The technique uses a new spherical Gaussian approximation of the BTDF allowing usage of pre-convolved environment map. The effects of complex geometry at the exting interface are approximated using a normal distribution function. These images are all rendered for increasing roughness in real-time $[\sim 140 \mathrm{~Hz}]$.

\section{Abstract}

We present an algorithm to render objects of transparent materials with rough surfaces in real-time, under distant illumination. Rough surfaces cause wide scattering as light enters and exits objects, which significantly complicates the rendering of such materials. We present two contributions to approximate the successive scattering events at interfaces, due to rough refraction : First, an approximation of the Bidirectional Transmittance Distribution Function (BTDF), using spherical Gaussians, suitable for real-time estimation of environment lighting using pre-convolution; second, a combination of cone tracing and macro-geometry filtering to efficiently integrate the scattered rays at the exiting interface of the object. We demonstrate the quality of our approximation by comparison against stochastic raytracing.

\section{Introduction}

We introduce a real-time algorithm to render translucency due to rough surfaces, such as frosted and misted glass. Translucent appearance can be attributed to a combination of two factors: surface scattering, for example in frosted glass due to its surface roughness, or volume scattering in participating media. In this paper we focus on the former and assume that the media are non-scattering. In the remainder of this paper we refer to such materials as being rough refractive.

In contrast to transparent materials with specular surface, handled by existing methods [Wyman 2005; Oliveira and Brauwers 2007], transparent materials with rough surfaces present the unique challenge that light is scattered in multiple directions as it enters and exits the object. The first consequence of these scattering events is that a light ray that enters at a single point of the surface will exit over an area that depends on the scattering properties of the material and the geometry of the object. The second consequence of light scattering in rough translucent objects is that the lighting computation involves the integration of light over multiple directions.

Our approach overcomes these difficulties by first observing that the scattering function used in previous work on rendering of rough transparent materials [Walter et al. 2007] can be well approximated by a spherical Gaussian. This allows us to replace the stochastic integration of lighting by a pre-integration, in the spirit of existing work on pre-convolution of environment lighting for real-time glossy reflections [Kautz et al. 2000; Ramamoorthi and Hanrahan 2002].

Our second contribution is a combination of cone tracing [Amanatides 1984] with geometry filtering [Han et al. 2007] to approximate the exiting rays, through a homogeneous material, due to scattering of a single incident ray. We approximate the geometry at the exiting area as the aggregate of the normals of the exiting surface intersected by the bounding cone of the refracted rays. We then convolve the resulting normal distribution function (NDF) with the material BTDF (Bidirectional Transmittance Distribution Function) to obtain a new scattering function that we use to query the preconvolved environment lighting.

To summarize, our paper makes the following contributions:

- we describe a method to render, in real time, rough refractive objects under all-frequency distant illumination (see Fig. 1).

- we introduce a new spherical Gaussian approximation of a microfacet BTDF to extend pre-convolution based methods to refractive materials.

- we propose an innovative formulation for approximating sequential refractions as convolutions involving the BTDF and the NDF at the exiting surface.

\section{Related work}

We review work on real time rendering of both reflection and refraction as well as related work in off-line rendering of refraction at rough surfaces. 


\begin{tabular}{|l|l|}
\hline $\mathbf{x}$ & Point \\
$\omega_{x}$ & Normalized direction \\
$\overrightarrow{\omega_{x}}$ & Un-normalized direction \\
$\omega_{o}$ & Outgoing direction \\
$\omega_{i}$ & Incoming direction \\
$\mathbf{n}$ & Normal \\
$\omega_{h_{r}}$ & Reflected half vector \\
$\omega_{t}$ & Transmitted half vector \\
$\eta_{i}$ & Index of refraction of the incident media \\
$\eta_{o}$ & Index of refraction of the refracting media \\
$f_{r}$ & BRDF (Bidirectional Reflectance Distribution Function) \\
$f_{t}$ & BTDF (Bidirectional Transmittance Distribution Function) \\
$\mathbf{p}$ & Intersection point on the first interface \\
$\mathbf{s}$ & Intersection point on the second interface \\
$S$ & Surface of integration on the second interface \\
$D$ & NDF(Normal Distribution Function) \\
$F$ & Fresnel function \\
$G$ & Geometric function for microfacet \\
$\Omega^{+}$ & Upper hemisphere \\
$\Omega^{-}$ & Lower hemisphere \\
$L\left(\omega_{o}\right)$ & Outgoing radiance \\
$L\left(\omega_{i}\right)$ & Incoming radiance \\
$\cdot$ & Dot product \\
$\chi$ \\
\hline
\end{tabular}

Table 1: Notations used in this paper.

Real-time reflection of distant illumination: In the case of glossy reflections, Kautz et al. [2000] and Ramamoorthi and Hanrahan [2002] have shown that for radially symmetric BRDFs, the contribution of distant incident illumination can be pre-integrated by means of a convolution of the environment map with the BRDFs. We extend these pre-convolution based methods for transparent materials with non-specular BTDFs.

Real-time refractions: Most existing work on real-time refractions focus on the special case of specular BTDFs, for which a single light ray travels through the object at each pixel [Wyman 2005; Oliveira and Brauwers 2007]. Recent methods on specular refractions handle complex light transport scenarios in real time, including non-homogeneous media and caustics [Sun et al. 2008; Ihrke et al. 2007; Cao et al. 2010]. While Heidrich et al. [1999] and Eisemann and Décoret [2006] address the rendering of rough refraction in real-time using pre-convolved environment maps, they approximate light transport inside the object with a single light ray. In contrast, our method relies on cone-tracing [Amanatides 1984] to efficiently account for multiple refracted rays inside the object.

BTDF models : Just as microfacet BRDFs model glossy reflections, a microfacet BTDF for rough refractions has been derived by Walter et al. [2007]. This model is expressed as (notation is shown in Table 1):

$$
\begin{aligned}
f_{t}\left(\omega_{h_{t}}, \mathbf{n}\right)= & \frac{\left|\omega_{i} \cdot \omega_{h_{t}}\right|\left|\omega_{o} \cdot \omega_{h_{t}}\right|}{\left|\omega_{i} \cdot \mathbf{n}\right|\left|\omega_{o} \cdot \mathbf{n}\right|} \\
& \frac{\eta_{o}^{2}\left(1-F\left(\omega_{o}, \mathbf{n}\right)\right) D\left(\omega_{h_{t}}, \mathbf{n}\right) G\left(\omega_{o}, \omega_{i}, \omega_{h_{t}}\right)}{\left(\eta_{i}\left(\omega_{i} \cdot \omega_{h_{t}}\right)+\eta_{o}\left(\omega_{o} \cdot \omega_{h_{t}}\right)\right)^{2}}
\end{aligned}
$$

where $\eta_{i}$ and $\eta_{o}$ are the indices of refraction (IOR) of the incident and refracting media respectively, $D$ is the normal distribution function (NDF) and $G$ is the geometric term that models effects such as masking and shadowing. Several choices are possible for the NDF D, such as the Beckmann and GGX [Walter et al. 2007] distribution. We use the Blinn-Phong model for its simplicity. The Blinn-Phong NDF is parametrized with $\kappa_{\gamma}^{D}$, the inverse roughness
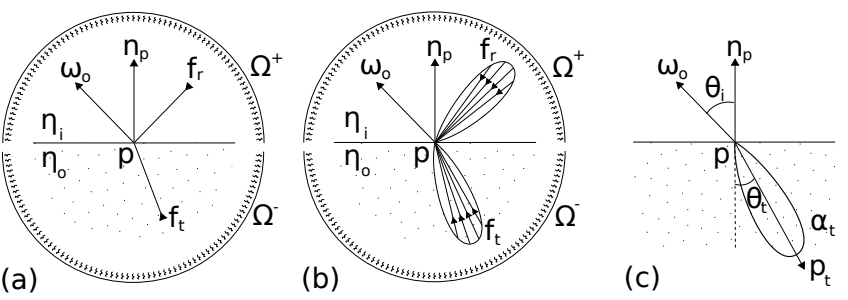

Figure 2: $B S D F$ and notation. (a) A specular BRDF $f_{r}$ and a specular BTDF $f_{t}$, (b) A glossy BRDF $f_{r}$ and a rough BTDF $f_{t}$, (c) Spherical Gaussian parametrization used for approximating the lobe of the refractive microfacet model.

(high values of $\kappa_{\gamma}^{D}$ yield sharp refractions) :

$$
D\left(\omega_{h_{t}}, \mathbf{n}\right)=\chi^{+}\left(\mathbf{n} \cdot \omega_{h_{t}}\right) \frac{\kappa_{\gamma}^{D}+2}{2 \pi}\left(\mathbf{n} \cdot \omega_{h_{t}}\right)^{\kappa_{\gamma}^{D}}
$$

This microfacet BTDF model is expressed according to the refractive half vector $\omega_{h_{t}}$, equivalent of the half vector for reflective models $\omega_{h_{r}}$ [Blinn 1977]. The refractive half vector is defined as :

$$
\omega_{h_{t}}=\frac{\omega_{h_{t}}}{\left\|{\overrightarrow{\omega_{h}}}_{t}\right\|}=-\frac{\eta_{o} \omega_{o}+\eta_{i} \omega_{i}}{\left\|\eta_{o} \omega_{o}+\eta_{i} \omega_{i}\right\|}
$$

When $\omega_{h_{t}}$ and the surface's normal $\mathbf{n}$ are collinear, $\omega_{i}$ and $\omega_{o}$ follow Snell's law:

$$
\eta_{i} \sin \theta_{i}=\eta_{o} \sin \theta_{o}
$$

Dai et al. [2009] propose a similar model for the particular case of thin surfaces, for which light transport in the object can be omitted. While these microfacet models can easily be integrated in a raytracer, the large number of samples required to handle rough materials is prohibitive for real-time applications. In this paper we identify that Walter's BTDF model is well approximated by a spherical Gaussian function, which allows us to pre-convolve environment maps for real-time rendering.

\section{Theory}

The exiting radiance $L\left(\mathbf{p}, \omega_{o}\right)$ at a point $\mathbf{p}$ on the surface of a transparent material, along the direction $\omega_{o}$, is the integral of the incoming radiance weighted by the reflectance function. For clarity, we separate the full scattering function into a $\operatorname{BRDF}\left(f_{r}\right)$, expressing scattering effects over the upper hemisphere $\left(\Omega^{+}\right)$, and a BTDF $\left(f_{t}\right)$, expressing scattering effects over the lower hemisphere $\left(\Omega^{-}\right)$. Both hemispheres, $\Omega^{+}$and $\Omega^{-}$are defined with respect to the tangent plane at $\mathbf{p}$.

$$
\begin{aligned}
L\left(\mathbf{p}, \omega_{o}\right)= & \int_{\Omega^{+}} L\left(\mathbf{p}, \omega_{i}\right) f_{r}\left(\omega_{o}, \omega_{i}\right) \mathrm{d} \omega_{i} \\
& +\int_{\Omega^{-}} L\left(\mathbf{p}, \omega_{i}\right) f_{t}\left(\omega_{i}, \omega_{o}\right) \mathrm{d} \omega_{i}
\end{aligned}
$$

For specular refraction, the BTDF $f_{t}$ is simply a Dirac along the direction given by Snell's law of refraction (see Figure 2a). For rough surfaces, the micro-geometry around point $\mathbf{p}$ defines the distribution $f_{t}$ (see Figure $2 \mathrm{~b}$ ).

Under our two-interface assumption, the exiting radiance at $\mathbf{p}$ is the result of successive scattering processes at two boundaries (see Figure 3). That is, ignoring the BRDF in Equation 5 and accounting 

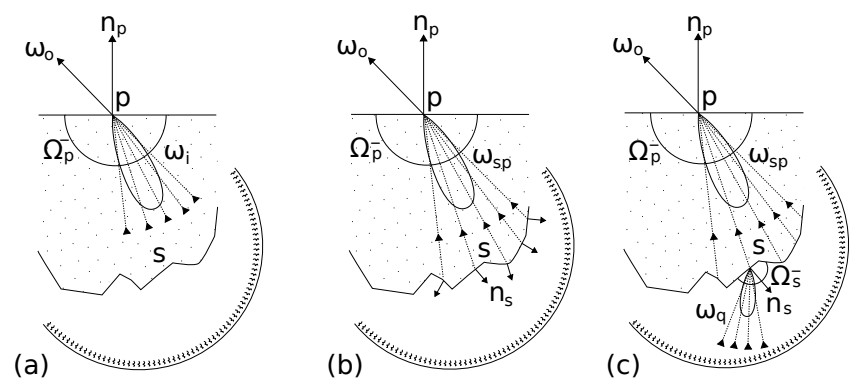

Figure 3: Refraction at two interfaces. (a) Integration of the BTDF at the first interface. (b) Stochastic integration of incident directions over the BTDF lobe at $\mathbf{p}$. (c) For each sampled direction in (b), integration over the BTDF lobe at $\mathbf{s}$ along which radiance is queried from the environment map.

for refraction at only two interfaces - entry and exit - on a homogeneous glossy object, the outgoing radiance at an entry point $\mathbf{p}$ can be expressed as an integral over the exiting surface $S$

$$
L\left(\mathbf{p}, \omega_{o}\right)=\int_{S} L\left(\mathbf{p}, \omega_{\mathbf{s p}}\right) f_{t}\left(\omega_{\mathbf{s p}}, \omega_{o}\right) J\left(\mathbf{n}_{\mathbf{p}}, \mathbf{n}_{\mathbf{s}}, \mathbf{p}, \mathbf{s}\right) \mathrm{d} \mathbf{s}
$$

where $\omega_{\mathbf{s p}}=\mathbf{s} \mathbf{p} /\|\mathbf{s} \mathbf{p}\|, \mathbf{n}_{\mathbf{p}}$ and $\mathbf{n}_{\mathbf{s}}$ are normals at $\mathbf{p}$ and $\mathbf{s}$ respectively, $J\left(\mathbf{n}_{\mathbf{p}}, \mathbf{n}_{\mathbf{s}}, \mathbf{p}, \mathbf{s}\right)$ is the Jacobian associated with the change of variables from solid angle to the surface $S$ and

$$
L\left(\mathbf{p}, \omega_{\mathbf{s p}}\right)=\int_{\Omega_{\mathbf{s}}^{+}} L\left(\mathbf{s}, \omega_{q}\right) f_{t}\left(\omega_{q}, \omega_{\mathbf{p s}}\right) \mathrm{d} \omega_{q} .
$$

\subsection{Refraction using convolution}

The primary challenge in real-time rendering of reflections and refractions for rough surfaces is rapid evaluation of the integral over $\Omega^{+}$and $\Omega^{-}$, which corresponds to a convolution of the incident illumination with a BSDF [Ramamoorthi and Hanrahan 2001]. Assuming distant lighting, and ignoring visibility, existing methods for real-time rendering of glossy reflections pre-compute the integral over $\Omega^{+}$by convolving the environment map with the BRDF $f_{r}$. This pre-convolution is independant of incident direction for radially symmetric BRDFs and the integral is evaluated at run time with a single look-up in the environment map in the direction of specular reflection.

We extend this pre-convolution approach to the case of refraction and pre-compute the integral over $\Omega^{-}$as a convolution of an environment map with the BTDF $f_{t}$. However, the pre-convolution approach requires a radially symmetric representation of the BTDF, which is not the case for the BTDF model of Walter et al. [2007]. We observe however that the BTDF response for a given incident angle and index of refraction is radially symmetric and can be well approximated by a spherical Gaussian, as illustrated in Figure 4. We express this spherical Gaussian representation of the BTDF as:

$$
f_{t}\left(\omega_{o}, \omega_{i}\right)=\alpha_{t} e^{\kappa_{t}\left(\mathbf{p}_{t} \cdot \omega_{i}-1\right)}
$$

where $\alpha_{t}$ is a scaling factor controlling the amplitude of the Gaussian lobe, $\kappa_{t}$ is an exponent controlling the width of the lobe, and $\mathbf{p}_{t}$ is the principal direction of the lobe (see Figure 2). These parameters are fitted for each incident angle $\theta_{i}$, each index of refraction $\eta$ and each BTDF exponent $\kappa_{\gamma}^{D}$ :

$$
T\left(\theta_{o}, \eta, \kappa_{\gamma}^{D}\right) \rightarrow\left(\alpha_{t}, \kappa_{t}, \mathbf{p}_{t}\right)
$$

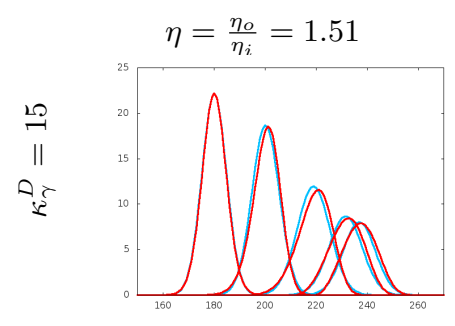

Angles

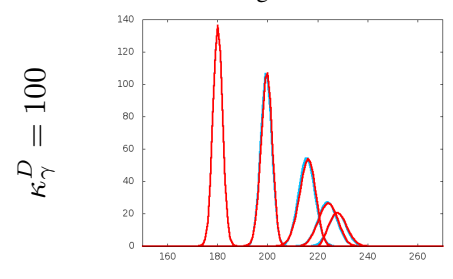

Angles

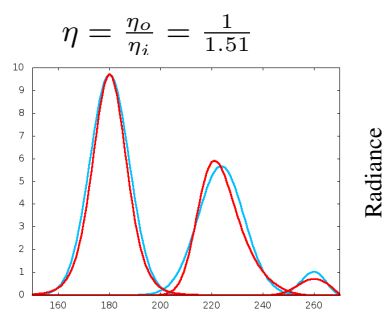

Angles

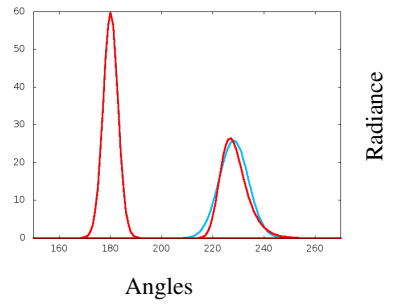

Angles
Figure 4: Comparison between the actual BTDF model (red) and our Gaussian approximation (blue) for different incident angles $(0,30,60,80$ and 88 degrees $)$, for two different values of roughness $\kappa_{\gamma}^{D}$. We show both values for light entering the material $(\eta=1.51)$ and for light leaving the material $\left(\eta=\frac{1}{1.51}\right)$. At the exiting interface, total internal reflection can happen, resulting in a smaller number of curves.
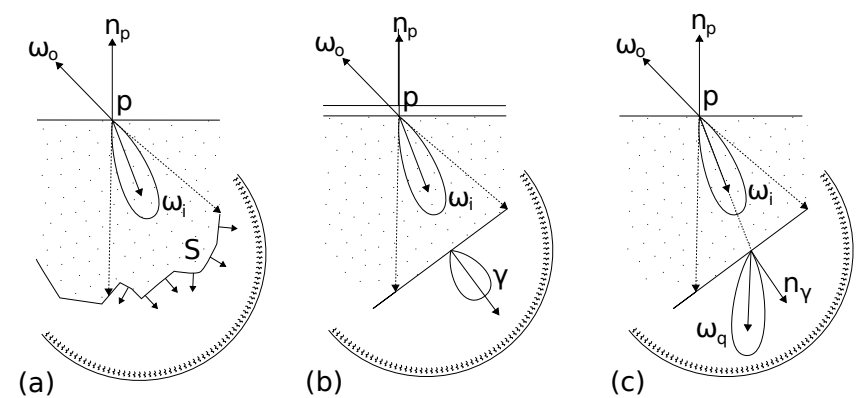

Figure 5: Approximated transport. (a) Transport from the first to the second interface with the actual geometry. (b) The geometry on the second interface is replaced by a single lobe NDF (mean + variance) (c) A new BTDF is derived from the NDF to include the local geometry.

For a given index of refraction $\eta$, we precompute the functions corresponding to the two interfaces: $T\left(\theta_{o}, \eta, \kappa_{\gamma}^{D}\right)$ and $T\left(\theta_{o}, 1 / \eta, \kappa_{\gamma}^{D}\right)$ and store them in 2D textures indexed by $\theta_{i}$ and $\kappa_{\gamma}^{D}$.

\subsection{Light transport as geometry filtering}

We now focus on light transport inside the object. As described by Equation 6 and Figure 3, light enters the transparent object at the first interface, is scattered by the BTDF and transported through the object, before finally exting at the second interface, where it is again scattered by the BTDF. Our goal is to find a good approximation of equation 6 for achieving real-time performance.

Our key idea is to replace the transport problem with a filtering problem (Figure 5): we extend the work of [Han et al. 2007] and represent the geometry intersected by the scattered rays at the second interface as a flat proxy enriched with a Normal Distribution Function (NDF). We then convolve this NDF with the BTDF to obtain a new BTDF that models the effect of both the transmittance function and the aggregated exiting geometry. 


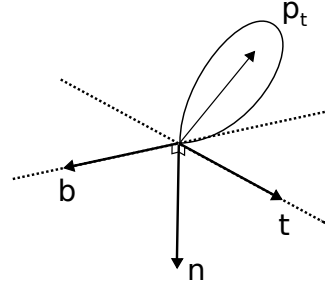

(a)

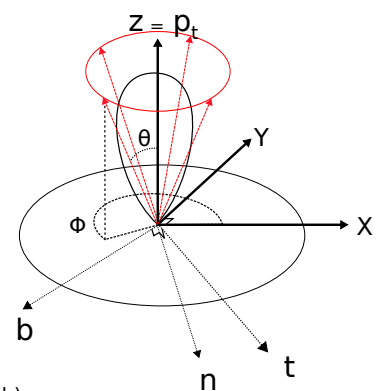

(b)
Figure 6: Lobe bounding : (a) Tangent frame in which the BTDF is expressed. (b) A tangent frame is built around the main axis of the BTDF, and 4 rays generated in spherical coordinates by using the bounding angle $\theta_{b}$ computed by equation 12 .

This approach is valid under three assumptions: (1) that all rays are parallel as they reach the exiting interface; (2) that the shape of the BTDF lobe is independent of the incident angle; and (3) that a single lobe NDF will be sufficient to represent the underlying geometry. While refraction is an important phenomenon, humans have difficulty perceiving or assessing the accuracy of results. Consequently, our model leads to plausible results with real-time frame rates despite the above assumptions. Our results, in Section 5 , demonstrates the efficacy of our model.

To compute the parameters of the resulting BTDF, we convolve the NDF of the geometry $\gamma$ (with an exponent $\kappa_{\gamma}^{G}$ ) with the original NDF of the BTDF, $D$ (with an exponent $\kappa_{\gamma}^{D}$ ). The result is a new $\mathrm{NDF}, D^{\prime}$, whose exponent $\kappa_{\gamma}^{D^{\prime}}$ is defined as:

$$
\kappa_{\gamma}^{D^{\prime}}=\frac{\kappa_{\gamma}^{G} \kappa_{\gamma}^{D}}{\kappa_{\gamma}^{G}+\kappa_{\gamma}^{D}}
$$

As shown in Figure 5c, we orient the proxy surface perpendicular to the average normal $n_{\gamma}$, and use it to refract the direction of the central lobe $\mathbf{p}_{t}$ of the first interface using the new BTDF.

\section{Algorithm}

Figure 7 describes the main steps of our algorithm, that we implement using three rendering passes. The first two passes compute geometric information about the scene, such as the NDF at the second interface at multiple scales and the range of depth covered by the back faces of the mesh. The third pass uses this information to approximate the transport of refracted rays inside the object with cone tracing. We finally convolve the NDF of the intersected geometry at the second interface with the BTDF and evaluate the illumination by a lookup in the pre-convolved environment map by the resulting distribution.

We describe here the pre-computation and the three rendering passes of our algorithm in more detail.

\subsection{Pre-computation}

Gaussian BTDF : We pre-compute the parameters $\theta_{t}, \alpha_{t}, \kappa_{t}$ of the Gaussian BTDF model for a regular sampling of incident angle $\theta_{i}$, index of refraction $\eta$ and exponent of the BTDF $\kappa_{\gamma}$. We fit by sampling the BTDF model using the importance sampling scheme given in [Walter et al. 2007] and then compute the scaling, mean and variance of the distribution.

While the resulting table could be stored in a 3D texture, a few 2D textures parameterized by $\theta_{i}$ and $\kappa_{\gamma}$ are often sufficient to store the parameters for the most common indices of refraction.
Environment Map : Similar to previous pre-convolution based methods [Kautz et al. 2000], we store the pre-convolved environment map as a MIP-map of 2D textures, where MIP-map levels contain the environment map convolved with spherical Gaussian functions of decreasing exponent $\kappa$.

\subsection{First and Second Pass : Geometry filtering}

In the first pass of our algorithm we render the normals and depth of back-facing geometry and store the result in off-screen buffers. We render the farthest back faces by setting up the depth test function to GL_GREATER in OpenGL, as in [Oliveira and Brauwers 2007]. In a second pass we recursively down-sample the back-face normal buffer to compute NDFs of the second interface at multiple scales. We use Toksvig's method [2005] to compute the downsampled normal buffer. In this method, the main direction of each NDF in the downsampled buffer is computed as the mean direction of the underlying normals. The variance $\sigma^{2}$ of the NDF is expressed as:

$$
\kappa_{\gamma}^{G}=\frac{2}{\sigma^{2}}=\frac{2(1-\|\overrightarrow{\mathbf{n}}\|)}{\|\overrightarrow{\mathbf{n}}\|}
$$

with $\overrightarrow{\mathbf{n}}$ the main direction of the NDF.

The normalized main direction $\overrightarrow{\mathbf{n}} /\|\overrightarrow{\mathbf{n}}\|$ is stored in the xy z components and the norm $\|\overrightarrow{\mathbf{n}}\|$ in the $\mathrm{w}$ component in order to interpolate them independently. This way, the variance of the NDF is smoothly interpolated unlike Toksvig's original method where variance can change rapidly between two texels if the NDF directions are very different.

We also compute the min and max value of the back-face depth buffer during the second pass using MIP-map reduction (For more details see [Oliveira and Brauwers 2007]). The depth buffer and its min and max value will then be used to trace rays inside the object using the ray marching algorithm of Oliveira and Brauwers [2007].

\subsection{Third Pass : Estimating macro-geometry NDF}

In the third pass, we render the front facing geometry and evaluate the final radiance at each pixel. To do so, we first compute the refraction at the first interface, and then trace a cone bounding the refracted rays inside the object. We finally use the NDF buffer to retrieve the NDF covered by the intersection of the cone with the second interface (pass 3 in Figure 7).

Refraction at the first interface: For each pixel, we use the incident angle $\theta_{i}$ relative to the local normal $\mathbf{n}$ and the BTDF exponent $\kappa_{\gamma}^{D}$ to read in the pre-computed texture values of $\alpha_{t 1} \kappa_{t 1}$ and $\mathbf{p}_{t 1}$ for the spherical Gaussian BTDF model.

Approximate cone tracing: We build a tangent frame where the $Z$ axis is oriented along the spherical Gaussian axis $\mathbf{p}_{t 1}$, as shown in figure $6 \mathrm{~b}$. We use this local frame to generate four rays bounding the spherical Gaussian lobe (Figure 6). We follow [Wang et al. 2009] and set the orientation $\theta$ of the rays in respect to the $Z$ axis such that the integral of the bounded lobe represents $75 \%$ of its total integral.

$$
\theta_{b}=\arccos \left((1-0.75)^{\frac{1}{\kappa_{t}+1}}\right)
$$

We use the ray marching algorithm of Oliveira and Brauwers [2007] to trace the four bounding rays inside the object. The four intersection points suggest the extent of the footprint of the refracted rays that intersect the second interface.

NDF estimation: The screen positions of the four intersection points define an axis-aligned bounding box of the pixels in the geometry buffers that are intersected by the refracted rays (pass 


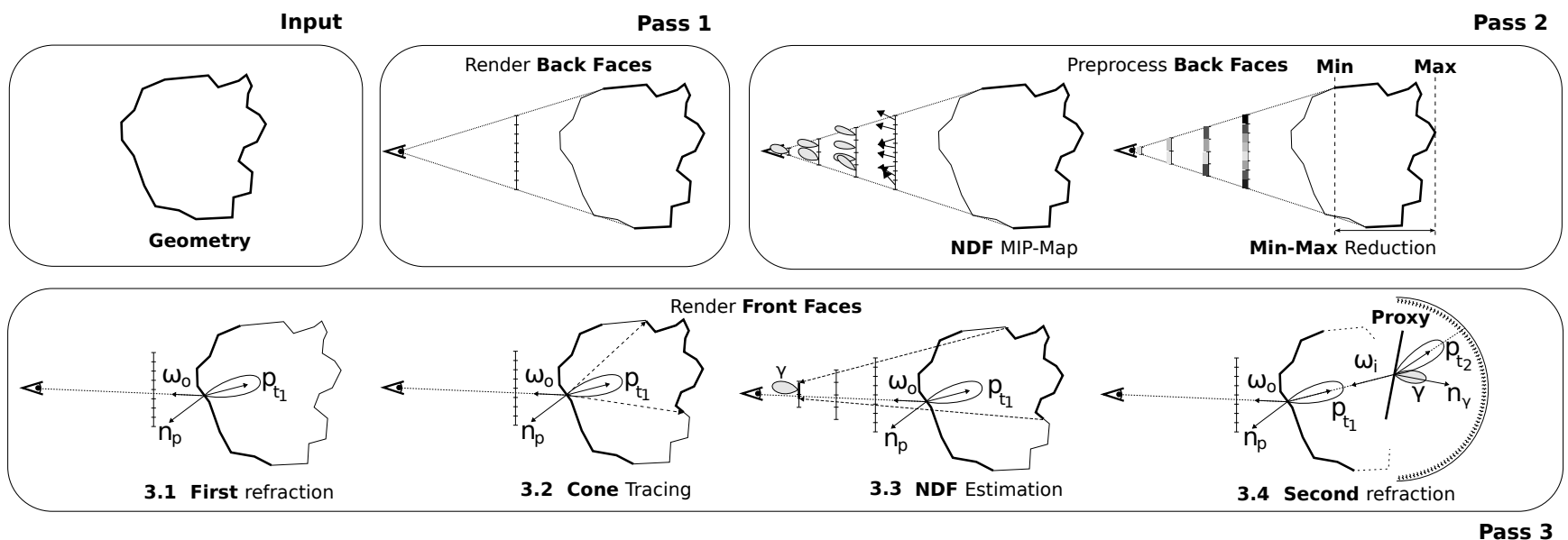

Figure 7: Overview of our algorithm. (Pass 1) During the first pass, the depths and the normals of back faces of the mesh are rendered into two separate buffers. (Pass 2) Based on the normal buffer, a NDF buffer is built, by estimating for each texel the mean direction and the variance. The min and max value of the depth buffer are also extracted. (Pass 3) During the last pass, the front faces of the mesh are rasterized. For each fragment, the parameters of the lobe at the first interface are extracted, and four rays are cast by ray marching in the depth texture for estimating its footprint. The NDF corresponding to this footprint is retrieved from the NDF buffer and is used as a proxy surface for the second refraction. The final radiance value is looked up into the pre-convolved environment map.

3.2 and 3.3 in Figure 7). We estimate the local texture coordinate derivatives $\left(\frac{\partial s}{\partial x}\right.$ and $\frac{\partial t}{\partial y}$ ) from this bounding box and use the OpenGL textureGrad function to look up the NDF value with an anisotropic sampler in the NDF buffer. The NDF texture has to be set up with anisotropic sampling for non-square bounding boxes.

Radiance computation: We use the NDF as a proxy surface for the second refraction (pass 3.4 in Figure 7). The angle $\theta_{i}$ between the central direction of the lobe on the first interface $\mathbf{p}_{t 1}$ and the NDF normal and the new exponent $\kappa_{\gamma}^{D^{\prime}}$ are used to look up into the parameter texture for retrieving the BTDF parameters $\alpha_{t 2}, \kappa_{t 2}$ and $\mathbf{p}_{t 2}$. Finally, those 3 parameters are used to look up the value of the incoming radiance in the pre-convolved environment map.

Total internal reflection: Correctly handling total internal reflection for objects with rough boundaries is expensive since it requires explicit tracing of individual rays. To avoid this, we adopt the popular solution introduced by Wyman [2005] and clamp the incident ray to the critical angle, at the upper bound, when the relative index of refraction is less than 1 .

\section{Results}

We have implemented the technique described in the paper in $\mathrm{C}++$ with OpenGL/GLSL. The images and videos have been rendered on an Intel Xeon $2.67 \mathrm{GHz}$ with a nVidia $260 \mathrm{GTX}$ graphics card. All images and videos are captured with a $512 \times 512$ resolution. The ground truth results are generated using a path tracer with importance sampling implemented using nVidia Optix [Parker et al. 2010]. Ground truth images are generated with 512 samples per pixel.

Since our technique does not require any precomputation on the mesh, we naturally support fully dynamic scenes.

\subsection{Experiments}

Figure 8 and Figure 10 show rough refractions through complex models in a comparison with ground truth. For fair comparison, images are rendered without managing total internal reflections. The scan line plot in Figure 8 illustrates the accuracy of our method for nearly convex objects.

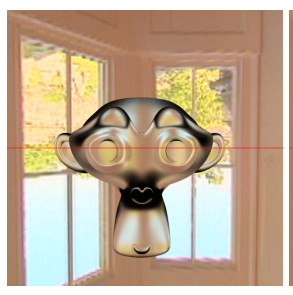

(a) Reference

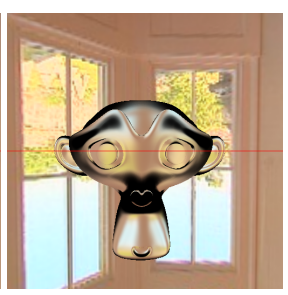

(b) Our model



(c) Line plot
Figure 8: Comparison with ground truth image without total internal reflection. Scan line plot of the values along the red line are shown. The graph shows a good fit between the ground truth and our technique. The arches of the monkey's eyebrow appear different using our model, since the model violates the convexity hypothesis.

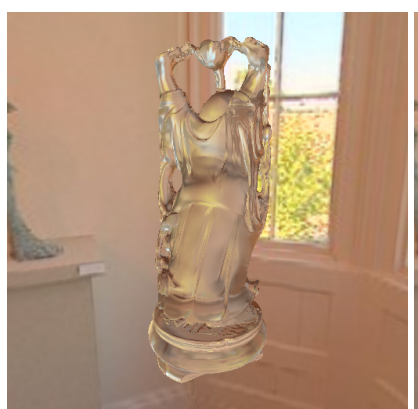

(a) Ground truth



(b) Our method
Figure 9: Comparison with ground truth with total internal reflection: While images have some differences, the result remains plausible.

Figure 9 shows comparisons of our method with ground truth with approximate total internal reflections. While some subtle differences can be noticed, our method produces plausible rough refractions despite approximate rendering of total internal reflections.

In Figure 11, we compare different methods for approximating light transport in the object. The left and middle images in Figure 11 approximate transport with a single ray using the method of Eisemann and Décoret [2006]. Our method better captures the low-pass filtering effect of the NDF and BTDF at the second interface. 


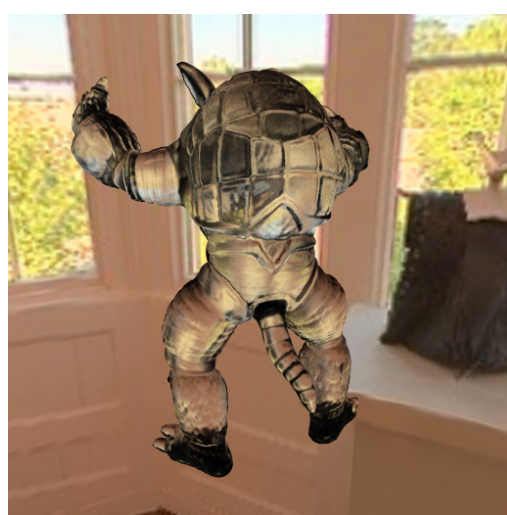

(a) Ground truth

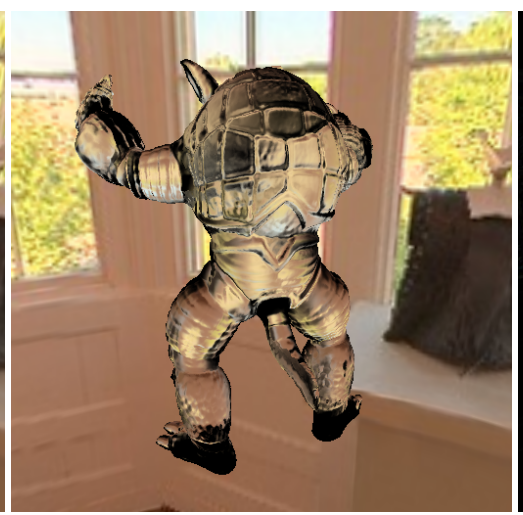

(b) Our method

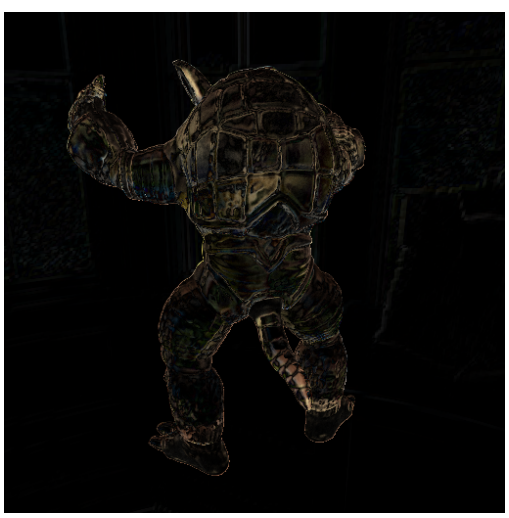

(c) Difference image

Figure 10: Comparison with ground truth without TIR. (Left) Ground truth. (Middle) Our method. (Right) The difference between the two images. Although our result is plausible, some artifacts are visible due to our assumptions (see Sec. 3.2).

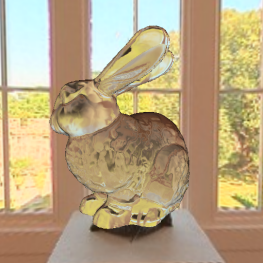

(a) Only depth without filtering

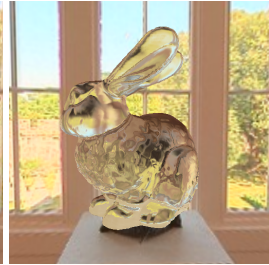

(b) Our BTDF

without filtering

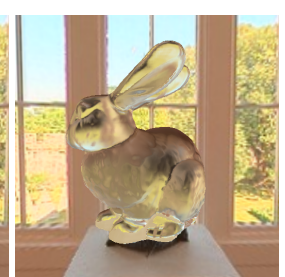

(c) Our BTDF
Figure 11: Comparison of transport methods: (Left) Specular transport with fixed mipmap level look up into the environment map based on depth traveled by the ray inside the object. (Middle) Specular transport with our spherical Gaussian model of the BTDF. (Right) Our approach, also accounting for the lobe tranport. Our technique accounts well for the low-pass filtering effect of the $B T D F$ on the geometry at the second interface.

Figure 1 shows the Bunny model with several BTDF exponents $\kappa_{\gamma}^{D}$ to illustrate the range of materials that can be achieved with our method. Our model supports modification of the BTDF's exponent $\kappa_{\gamma}^{D}$ in real-time, allowing artists to adjust the glossiness of the refractions to obtain a desired appearance.

Figure 12 summarizes the performances of our method. While our method for rendering rough materials is on average two times slower than rendering of specular materials [Oliveira and Brauwers 2007], we achieve a speed up of several orders of magnitude compared to ground truth raytracing for comparable image quality. Since our technique is mainly excuted in a fragment shader, we indicate the number of pixels covered by the geometry in addition to the number of triangles. The graph in Figure 12 illustrates the rendering cost of each pass of our algorithm, as well as the rendering cost for purely specular materials [Oliveira and Brauwers 2007].

\subsection{Limitations}

Spatially-varying BTDFs: Our method can handle spatiallyvarying BTDFs at the first interface as specified by a texture map. However, our light transport approximation in the object is not compatible with spatially-varying BTDFs at the second interface.

Screen-space filtering: Since we rely on a screen-space algorithm to estimate the NDF at the second interface, our method is restricted to geometry that lies inside the view frustum.

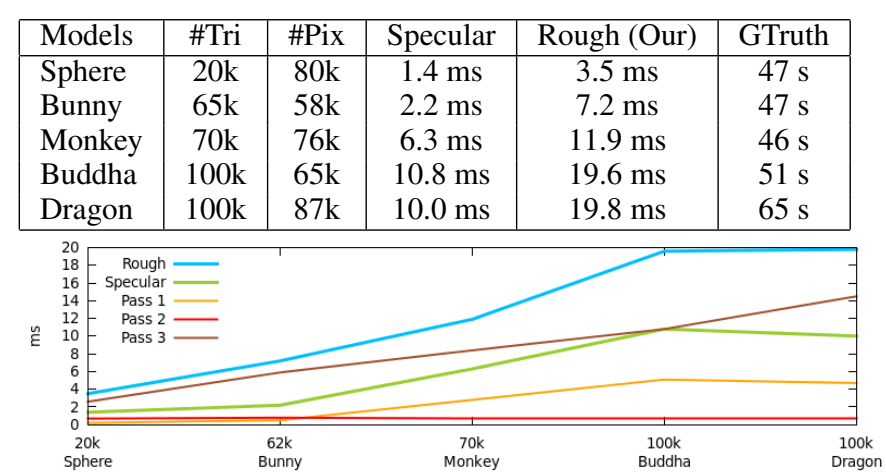

Figure 12: This table shows the performances of our methods according to the number of triangles \#Tri and the number of pixels \#Pix covered by the geometry. We compare the timings of our method to a GPU specular refraction algorithm and to the ground truth rendering generated with Optix. Ground truth timings are given for 512 samples per pixel.

\section{Conclusion}

We have presented a new algorithm for rendering transparent objects with rough surfaces. Our algorithm runs in real-time, and renders plausible images. Our algorithm is based on two contributions: a new formulation for the scattering function at the interface, and a combination of cone tracing and geometry filtering to approximate the exiting rays at the exiting interface.

Our new formulation for the scattering function at the interface is an approximation of the actual BTDF [Walter et al. 2007], using spherical Gaussians. The approximation is better suited for convolution with the incoming light and the geometry.

Our combination of cone-tracing with geometry filtering allows us to approximate the light transport inside the transparent object, aggregating the normals over the area intersected by the bounding cone of the refracted rays. We then convolve the resulting normal distribution function with the BTDF of the surface to obtain a new scattering function, that we use to query the pre-convolved environment lighting.

Our method runs in real-time, can handle complex objects and accounts for the integration that happens due to scattering at the exiting interface. In future work, we would like to extend this approach to handle spatially varying BTDFs and total internal reflections. Our approach for filtering the normals at the exiting interface 


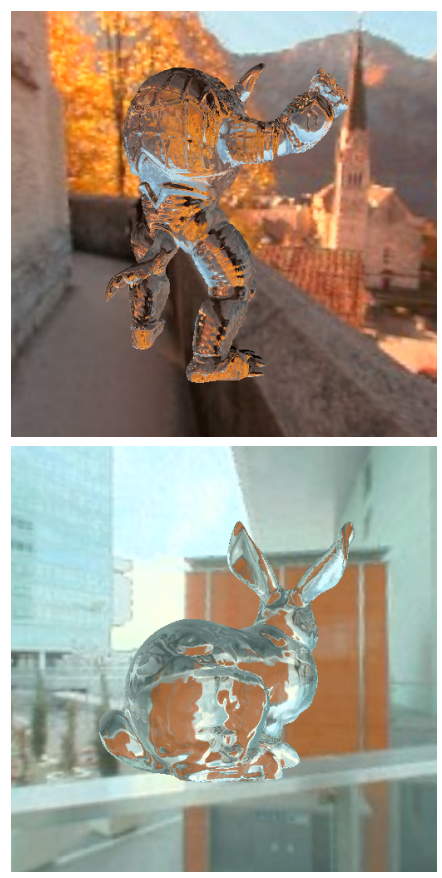

(a) $\kappa_{\gamma}^{D}=20000$
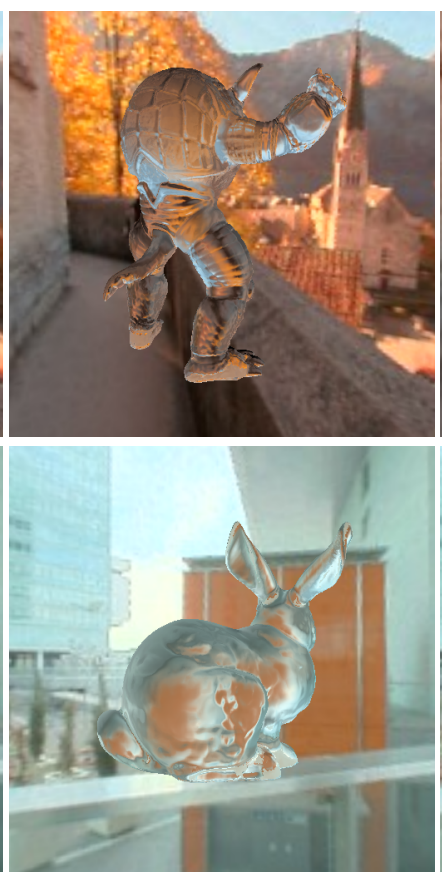

(b) $\kappa_{\gamma}^{D}=90$
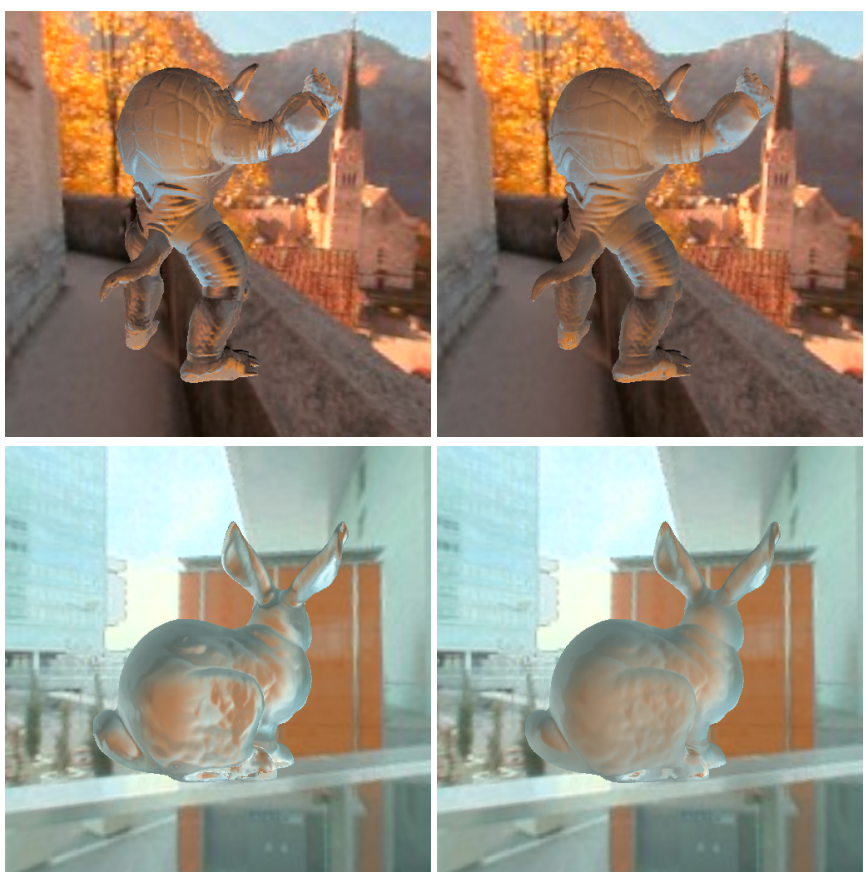

(c) $\kappa_{\gamma}^{D}=24$

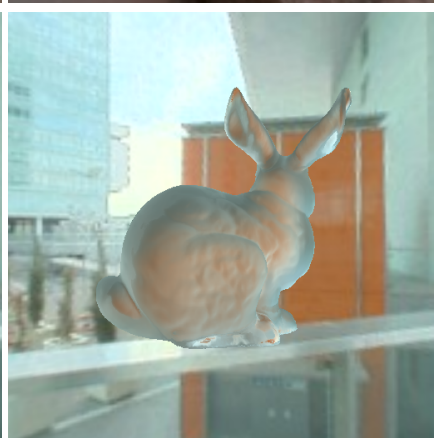

(d) $\kappa_{\gamma}^{D}=6.4$

Figure 13: Additional results with Bunny and Armadillo models under various environment maps for different roughness.

could also be applied for indirect illumination of complex reflective objects.

\section{Acknowledgments}

This work was supported in part by NSF grant 0924968 , ANR grant ANR-07-MDCO-001, and the ExploraDoc program. We also acknowledge additional support from NSF grant 1011832, NVIDIA through a professor partnership award and graphics cards, and equipment and funding from Intel, Adobe and Pixar. The environment map used in this paper were provided by Bernhard Vogl (http://dativ.at/lightprobes/). The models used in this paper come from the Stanford 3D scanning respository, except the monkey model which comes from the Blender project.

\section{References}

Amanatides, J. 1984. Ray tracing with cones. Computer Graphics (SIGGRAPH '84) 18, 3, 129-135.

BLINN, J. F. 1977. Models of light reflection for computer synthesized pictures. Computer Graphics (SIGGRAPH '77) 11, 3, 192-198.

CAO, C., ReN, Z., GUO, B., AND ZHou, K. 2010. Interactive Rendering of Non-Constant, Refractive Media Using the Ray Equations of Gradient-Index Optics. Computer Graphics Forum (EGSR proceedings) 29, 4, 1375-1382.

DAi, Q., WANG, J., Liu, Y., SnYder, J., Wu, E., AND Guo, B. 2009. The dual-microfacet model for capturing thin transparent slabs. Computer Graphics Forum 28, 7, 1917-1925.

Eisemann, E., AND DÉcoret, X. 2006. Fast scene voxelization and applications. In Symposium on Interactive 3D Graphics and Games (I3D), 71-78.

Han, C., Sun, B., Ramamoorthi, R., And Grinspun, E. 2007. Frequency domain normal map filtering. ACM Transactions on Graphics (SIGGRAPH 2007) 26, 3, 28.

Heidrich, W., Lensch, H., Cohen, M. F., And Seidel, H.-P. 1999. Light field techniques for reflections and refractions. In Eurographics Symposium on Rendering.
Ihrke, I., Ziegler, G., Tevs, A., Theobalt, C., Magnor, M., And SEIDEL, H.-P. 2007. Eikonal rendering: Efficient light transport in refractive objects. ACM Transactions on Graphics (Siggraph'07) 26, 3 (Aug.), 59.

Kautz, J., VÁzquez, P.-P., Heidrich, W., AND Seidel, H.-P. 2000. Unified approach to prefiltered environment maps. In Eurographics Workshop on Rendering, 185-196.

Oliveira, M. M., AND BRAuwers, M. 2007. Real-time refraction through deformable objects. In Symposium on Interactive 3D Graphics and Games (I3D), 89-96.

Parker, S. G., Bigler, J., Dietrich, A., Friedrich, H., HobeRock, J., Lueb Ke, D., McAllister, D., McGuire, M., Morley, K., RoBison, A., AND STICH, M. 2010. Optix: a general purpose ray tracing engine. ACM Transactions on Graphics 29, 4, 1-13.

RAMAMOORThi, R., AND HANRAhan, P. 2001. A signal-processing framework for inverse rendering. In SIGGRAPH '01, 117-128.

Ramamoorthi, R., AND Hanrahan, P. 2002. Frequency space environment map rendering. ACM Transactions on Graphics ( $S I G$ GRAPH '02) 21, 3, 517-526.

Sun, X., Zhou, K., Stollnitz, E., Shi, J., And Guo, B. 2008. Interactive relighting of dynamic refractive objects. ACM Transactions on Graphics (SIGGRAPH '08) 27, 3, 1-9.

ToKsvig, M. 2005. Mipmapping normal maps. journal of graphics, GPU, and game tools 10, 3, 65-71.

WALter, B., Marschner, S. R., Li, H., AND TORRANCE, K. E. 2007. Microfacet models for refraction through rough surfaces. In $E u$ rographics Symposium on Rendering.

Wang, J., Ren, P., Gong, M., Snyder, J., And Guo, B. 2009. Allfrequency rendering of dynamic, spatially-varying reflectance. ACM Transactions on Graphics 28, 5.

WyMAN, C. 2005. An approximate image-space approach for interactive refraction. ACM Transactions on Graphics (SIGGRAPH 2005) $24,3,1050-1053$. 